\title{
Fiber-optic chemical sensing with Langmuir-Blodgett overlay waveguides
}

\author{
Damien Flannery, Stephen W. James, Ralph P. Tatam, and Geoffery J. Ashwell
}

\begin{abstract}
Fiber-optic chemical sensing has been demonstrated with a side-polished single-mode optical fiber, evanescently coupled to chemically sensitive Langmuir-Blodgett (LB) overlay waveguides. The sensors exhibit a channel-dropping response centered on a wavelength that is dependent on the thickness and the refractive index of the overlay waveguide. It has been shown that $\mathrm{pH}$-sensitive organic dyes proved to be suitable materials for the formation of an overlay waveguide whereas LB deposition provides the required thickness control. A theoretical model of the sensor response, based on the Kramers-Kronig relations and phase matching of the guided modes within the optical fiber and overlay waveguide, shows good agreement with experimental results. (C) 1999 Optical Society of America

OCIS codes: $060.0060,060.2370$.
\end{abstract}

\section{Introduction}

Fiber-optic sensors are becoming increasingly more important, particularly in the field of chemical sensing, ${ }^{1}$ because of the advantages they offer compared with conventional electrical sensors. The most obvious is their immunity to electromagnetic interference. However, extremely high bandwidths, small size, the possibility of multiplexing several sensors for truly and quasi-distributed sensing, and the reduced risk of ignition when sensing volatile species also make them attractive.

Fiber-optic chemical sensors that use evanescent absorption techniques for such chemicals as methane gas have been reported. In this type of sensor, one can access part of the evanescent field guided by the fiber by reducing its diameter through heating and stretching $^{2}$ or by using D-shaped fiber. ${ }^{3}$ A drawback is that they are capable only of sensing chemical species that exhibit absorption at the wavelength of available laser sources. However, the fibers can be coated with materials that, with a change in a particular optical property such as fluorescence, ${ }^{4}$ can be interrogated at a suitable wavelength. In this way, many more chem-

All the authors are with the Centre for Photonics and Optical Engineering, School of Mechanical Engineering, Cranfield University, Cranfield, Bedford MK43 OAL, UK. D. Flannery is with the Optical Sensors Group and the Nanomaterials Group. S. W. James and R. P. Tatam (r.p.tatam@cranfield.ac.uk) are with the Optical Sensors Group. G. J. Ashwell is with the Nanomaterials Group.

Received 3 March 1999; revised manuscript received 23 August 1999.

0003-6935/99/367370-05\$15.00/0

(C) 1999 Optical Society of America ical species can be sensed. One significant advantage to all evanescent-wave sensors is their fast response but, inasmuch as only the light present in the evanescent field is used, their sensitivity can be limited.

In a previous publication ${ }^{5}$ we demonstrated a fiberoptic $\mathrm{pH}$ sensor by using a side-polished single-mode optical fiber evanescently coupled to a single-mode planar waveguide overlay. Light is coupled from the fiber core into the overlay waveguide at a wavelength that is dependent on the refractive index and the thickness of the overlay waveguide. If the overlay material changes its refractive index in the presence of a chemical species, then the wavelength at which the light is coupled into the overlay also changes. As most of the light guided by the fiber is coupled into the chemically sensitive overlay waveguide, such sensors exhibit a high sensitivity while retaining the fast response times associated with other evanescentwave chemical sensors. Furthermore, as the materials used to form the overlay waveguide are organic, it is possible to tailor the properties.

Here the possibility of chemical sensing by use of such technology is investigated further. In particular the use of a new chemical material, a substituted merocyanine dye, has been used to form the Langmuir-Blodgett (LB) overlay. This gives improved properties, for example, redshifting the absorption maximum in such a way that the channeldropping wavelength would shift further into the infrared. The Kramers-Kronig relations have been used successfully to model the nonlinear $\mathrm{pH}$ sensor response. This differs substantially from the original study in which the combination of material and wavelength resulted in a linear response. 


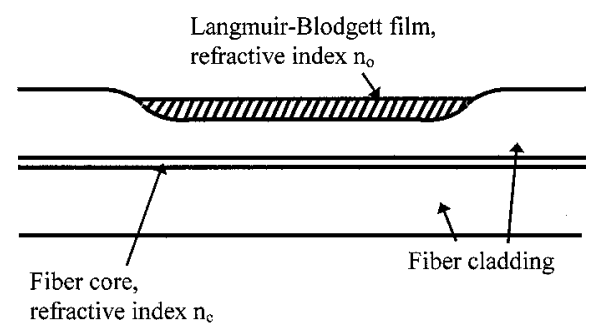

Fig. 1. Schematic diagram of a fiber-optic chemical sensor.

\section{Sensor Design}

The chemical sensors reported in this paper are based on a previously reported design for an in-line fiberoptic channel-dropping filter ${ }^{6}$ (Fig. 1). Such devices consist of a side-polished single-mode optical fiber, evanescently coupled to a single-mode planar overlay waveguide, deposited directly as a LB film onto the polished region of the fiber. Light is coupled from the fiber core into the overlay at a particular coupling wavelength $\lambda_{m}$. This occurs when the two waveguides, the fiber core and the planar overlay, become phase matched and can be calculated by inserting the phase-matching condition into the eigenvalue equation for the zeroth-order mode of a three-layer, asymmetric, isotropic slab waveguide ${ }^{7}$ :

$$
\frac{2 \pi d}{\lambda_{m}}\left(n_{o}^{2}-n_{\mathrm{eff}}^{2}\right)^{1 / 2}=\Phi_{s}+\Phi_{\mathrm{cl}},
$$

where $n_{o}$ is the overlay refractive index, $n_{\text {eff }}$ is the effective refractive index of the fiber mode, $d$ is the thickness of the overlay waveguide, and $\Phi_{s}$ and $\Phi_{\mathrm{cl}}$ are polarization-dependent phase change terms at the overlay-superstrate and overlay-fiber cladding boundaries, respectively. The dependence of the coupling wavelength on the thickness and on the refractive index of the overlay material can be seen from Eq. (1).

After the sensor has been fabricated, only the refractive index of the overlay affects the coupling wavelength, but, as the overlay is being deposited, it is the thickness of the overlay that defines the coupling wavelength. In practice, the thickness of the overlay is critical and therefore LB deposition is ideally suited because it allows extremely accurate control. To polish the optical fiber, a small section of the protective jacket $(\sim 25 \mathrm{~mm})$ is removed and some of the exposed cladding is polished away by use of an annular polishing technique. ${ }^{8}$ The amount of cladding removed is measured by an oil-drop test ${ }^{9}$ and is expressed as a percentage drop in the transmitted power at a single wavelength when refractive-index oil $(n=1.464)$ is added to the polished region. After a fiber has been polished to the required depth, one can obtain the planar overlay waveguide of a suitable chemically sensitive material by LB deposition.

\section{Langmuir-Blodgett Deposition}

The thickness of the overlay waveguide defines the coupling wavelength of the sensor for a given ma-

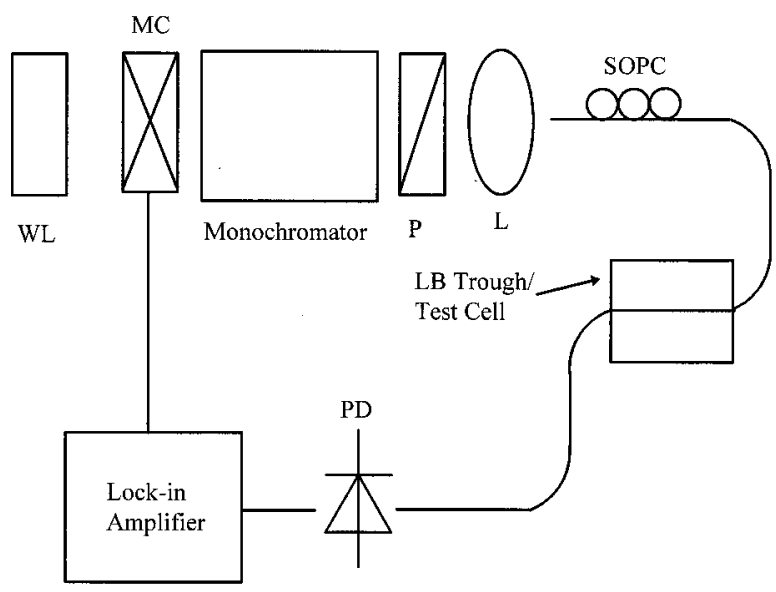

Fig. 2. Experimental apparatus: WL, white-light source; MC, mechanical chopper; P, polarizer; L, 20× microscope objective; SOPC, state of polarization controller.

terial refractive index, and the LB technique ${ }^{10}$ facilitates the required tolerances by permitting layer-bylayer control of the floating monolayer (2-3 nm thick). In this case, we performed LB deposition on a Nima Technology LB trough and we accomplished continuous monitoring of the growth of the overlay waveguide by coupling white light into a length of single-mode optical fiber through a monochromator, polarizer, and microscope objective (Fig. 2). A fiber-optic state of polarization controller was used to ensure TM polarization in the fiber as, from Eq. (1), the phase-matching wavelength is polarization dependent. We monitored the transmission by using a photodiode and a lock-in amplifier. With this system, it is possible to cease deposition at an appropriate thickness for phase matching at a given wavelength.

A substituted merocyanine dye was used as the chemically sensitive overlay waveguide material (Fig. 3) because it is $\mathrm{pH}$ sensitive through the terminal oxygen atom that can be reversibly protonated. The dye exhibits two distinct states, protonated and unprotonated, each of which has different absorption spectra (Fig. 4), resulting in a significant refractiveindex change after switching, the magnitude of the change being dependent on the change in absorption. The process is partially reversible when the film is initially exposed to $\mathrm{HCl}$ and $\mathrm{NH}_{3}$, but when it is repeatedly exposed the process reversibly changes between different intermediate states.

\section{Experimental Results}

A length of optical fiber (Spectran SMC-A0780B, 780-nm operating wavelength) was polished as de-

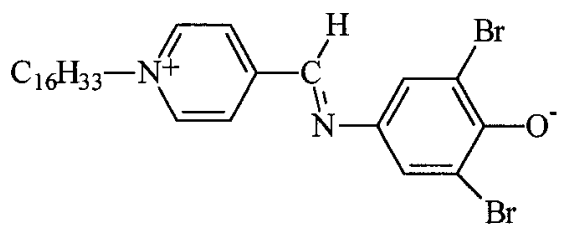

Fig. 3. Molecular structure of the substituted merocyanine dye. 




Fig. 4. Absorption spectra of a 50-layer film of the merocyanine dye before and after exposure to $\mathrm{HCl}$ vapor.

scribed above to give a coupling strength of $19 \%$ at the oil refractive index of 1.464 . We deposited 178 layers of the merocyanine dye onto the polished region at a constant surface pressure of $36 \mathrm{mN} \mathrm{m}^{-1}$, with the first six layers deposited at a rate of $3 \mathrm{~mm}$ $\min ^{-1}$ and the remainder at $20 \mathrm{~mm} \mathrm{~min}{ }^{-1}$. This gave the sensor a coupling wavelength of $900 \mathrm{~nm}$ which, when exposed to $\mathrm{HCl}$ vapor followed by $\mathrm{NH}_{3}$, shifted to $775 \mathrm{~nm}$ as shown in Fig. 5 .

We positioned the sensor in a temperaturecontrolled test cell by using the experimental setup shown in Fig. 2. Solutions in 7-mL portions and differing in $\mathrm{pH}$ were successively introduced to the test cell and allowed to reach a constant temperature of $25 \pm 0.05{ }^{\circ} \mathrm{C}$. The lock-in amplifier time constant was set at $100 \mathrm{~ms}$ to provide a steady signal, and the monochromator was then stepped through a predetermined wavelength range at a rate of $0.4 \mathrm{~nm} \mathrm{~s}^{-1}$. The resulting fiber transmission was recorded on the lock-in amplifier at a sampling rate of $8 \mathrm{~Hz}$ and normalized to the spectral characteristics of a length of unpolished optical fiber (Fig. 6).

As the sensor reported within this paper relies on changes in refractive index of the overlay waveguide material, we investigated its response by using a theoretical model based on the Kramers-Kronig relations, which is described in Section 5.

\section{Theoretical Model}

The complex refractive index of an absorbing medium can be expressed as the sum of a real part (refractive



Fig. 5. Normalized TM transmission of the sensor after exposure to $\mathrm{HCl}$ vapor and an ammonia solution.

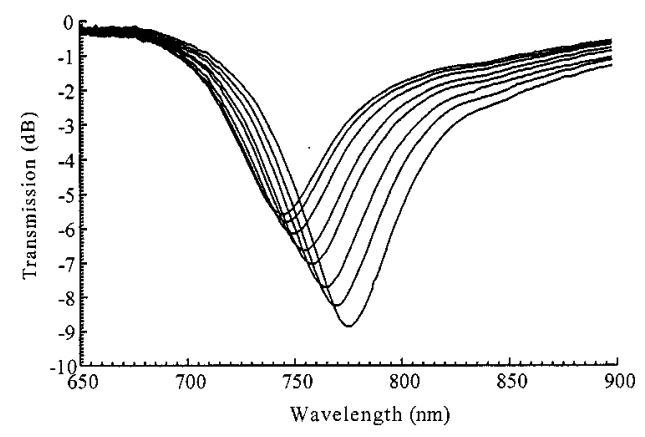

Fig. 6. Normalized TM sensor transmission in solutions of, from left to right, $\mathrm{pH} 11.60,11.83,12.09,12.39,12.64,12.80,13.02$, and 13.23.

index $n_{r}$ ) and an imaginary part (extinction coefficient $n_{i}$ ) as shown in Eq. (2):

$$
n_{\text {complex }}=n_{r}+i n_{i}
$$

The relationship between $n_{r}$ and $n_{i}$ can be described by the Kramers-Kronig relationship ${ }^{11}$ as follows:

$$
\begin{aligned}
& n_{r}(v)=n_{r \infty}+\frac{2}{\pi} \int_{0}^{+\infty} \frac{s n_{i}(s)}{s^{2}-v^{2}} \mathrm{~d} s, \\
& n_{i}(v)=-\frac{2 v}{\pi} P \int_{0}^{+\infty} \frac{n_{r}(s)}{s^{2}-v^{2}} \mathrm{~d} s,
\end{aligned}
$$

where $n_{r \infty}$ is the refractive index of the material at a wavelength far from the absorption band, $P$ is the principal value of the integral, and $v$ is the wave number at which the refractive index is calculated due to the material absorption over the range of wave numbers defined by $s$. Furthermore, the extinction coefficient for the material is related to the bulk absorption coefficient $\alpha$ by

$$
n_{i}=\alpha \lambda / 4 \pi
$$

This can be calculated from the measured absorbance $(A)$ and material thickness $(l)$ by

$$
\alpha=\log _{10}(e) A / l \text {. }
$$

It is now possible to calculate the refractive index of the overlay material directly from its absorption spectrum at all wavelengths with Eqs. (3), (5), and (6). However, to perform the integration in Eq. (3), it is necessary to describe the absorption spectrum as a continuous function. These were studied by fitting to Lorentzian and Gaussian functions and their combinations as commonly used in the field of molecular spectroscopy. ${ }^{12}$

We performed the fitting to the measured absorption spectra by using the graphics package Microcal Origin $3 \mathrm{D}$. The most appropriate fitting function for the spectra of the overlay material, when plotted in terms of wave numbers, consisted of the sum of two Gaussian functions. An example of an absorption spectrum and the fitted function can be seen in Fig. 7. The next stage in the modeling is to take the function generated 


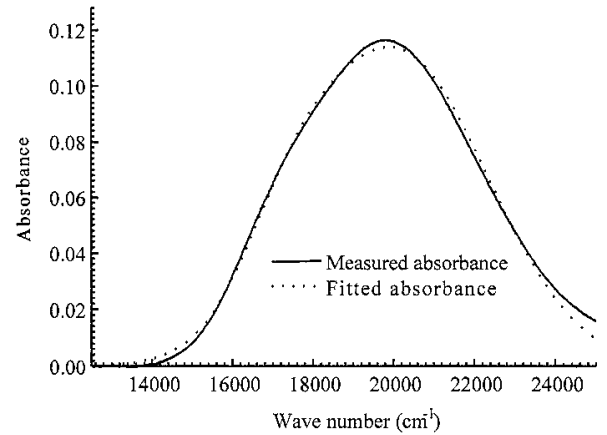

Fig. 7. Plot of the absorption spectrum of 50 layers of the merocyanine dye and the Gaussian fit derived from Microcal Origin 3D.

by the fitting process and calculate the imaginary part of the refractive index by use of Eqs. (5) and (6). A thickness of $2 \mathrm{~nm}$ per layer was assumed and $n_{r \infty}$ was estimated as 1.4. The integral in Eq. (3) was then calculated to give the real part of the refractive index as a function of wave number. To simplify the integral in Eq. (3) it was noted that the function $s /\left(s^{2}-v^{2}\right)$ decays rapidly to either side of the wave number $s$. In fact it was found that this function contributed no significant part to the integral at wave numbers beyond $9000 \mathrm{~cm}^{-1}$ either side of $s$ for all the values of $s$ used in the following model. We used 30,000 points for numerical evaluation of the integral ${ }^{13}$ with 100 $\mathrm{cm}^{-1}$ steps in the wave number. A plot of the fitted function, shown in Fig. 7, along with the resultant refractive-index plot from the modeling can be seen in Fig. 8. The refractive index rises sharply close to the absorption maximum while going through the wellknown anomalous dispersion regime.

To model the effect of the refractive-index changes that are due to $\mathrm{pH}$, the spectra of a 50-layer LB film on a planar substrate were investigated (Fig. 9). The glass slide was exposed to $\mathrm{HCl}$ vapor and then Gaussian functions were fitted to the measured absorption spectra. We calculated the refractive index of the LB film as a function of wavelength by using the thickness of $2 \mathrm{~nm}$ per layer (Fig. 10). Note that the results shown in Fig. 10 are refractive-index changes as opposed to an absolute value because $n_{r \infty}$



Fig. 8. Plot of the absorption spectrum created by the fit in Fig. 7 and its corresponding material dispersion curve as calculated by the Kramers-Kronig model, assuming that $n_{r \infty}=1.4$.

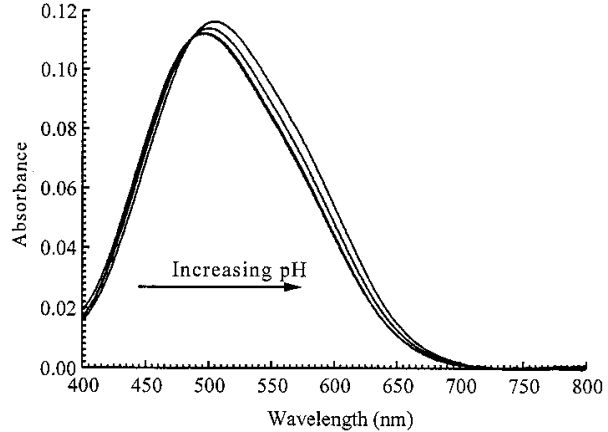

Fig. 9. Plot showing shifts in the absorption spectra of 50 layers of the merocyanine dye in solutions of $\mathrm{pH} 11.56,11.98,12.60$, and 13.23 .

is not known accurately. However it is possible to predict the changes in sensor coupling wavelength.

To predict the shifts in sensor coupling wavelength under the conditions described, it is necessary to analyze the sensor's coupled waveguide structure. Coupling between the two waveguides occurs when the effective refractive index of the overlay guided mode equals that of the optical fiber mode. The effective refractive index of the former depends not only on the refractive index of the overlay material but also on the refractive index of the ammonia solution. Furthermore, with such asymmetric, thin planar waveguides, waveguide dispersion is also an important factor. To analyze this complex structure, we used a numerical model based on the transfer matrix approach for the study of multilayer thin-film structures developed by Ghatak et al. ${ }^{14}$ This allows for the calculation of effective refractive indices of bound modes within complex dielectric waveguides.

To calculate the wavelength at which the overlay becomes phase matched to the optical fiber it is necessary to model the dispersion within each waveguide separately. As mentioned above, the refractive index of the ammonia solution affects the dispersion of the overlay waveguide. Therefore it is necessary to measure its refractive index as a function of the $\mathrm{pH}$ by use of an Abbé refractometer illuminated by a tunable Ti: sapphire laser, the results of which can be seen in Fig. 11. From these results, coupled with the material dispersion results shown in Fig. 10, it is possible to

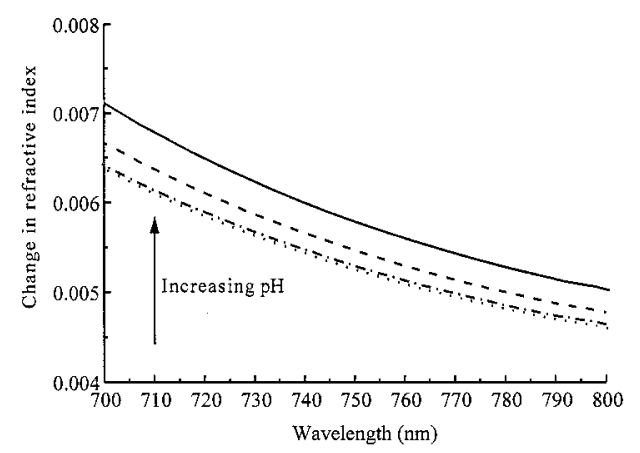

Fig. 10. Material dispersion curves calculated from the absorptions shown in Fig. 9. 


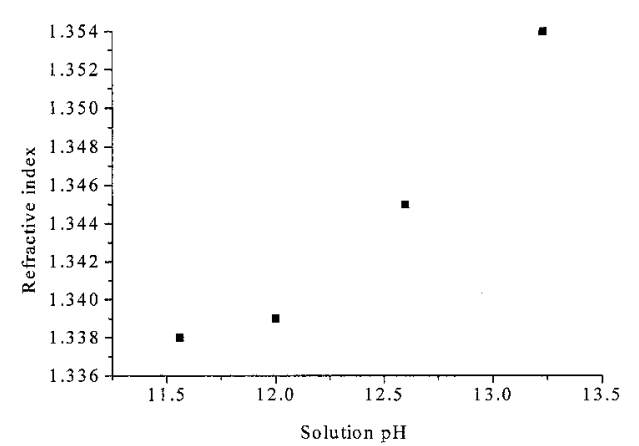

Fig. 11. Refractive index of ammonia solutions of $\mathrm{pH} 11.56,11.98$, 12.60 , and 13.23 measured at wavelengths of $745,747.5,757.5$, and $775 \mathrm{~nm}$, respectively.

calculate the overall dispersion curves for the overlay waveguide with the matrix method. These results are shown in Fig. 12 along with the dispersion curve for the optical fiber. Phase matching occurs when the dispersion curve at a given $\mathrm{pH}$ value crosses that of the optical fiber, and these wavelengths can easily be obtained from Fig. 12. A plot of predicted phasematching wavelengths, along with the experimental results shown in Fig. 6, can be seen in Fig. 13. It should be noted that, to predict the phase-matching wavelength of the sensor, it is necessary to have an absolute value of the overlay material refractive index as opposed to the relative changes shown in Fig. 10 . We obtained this absolute value by calculating the

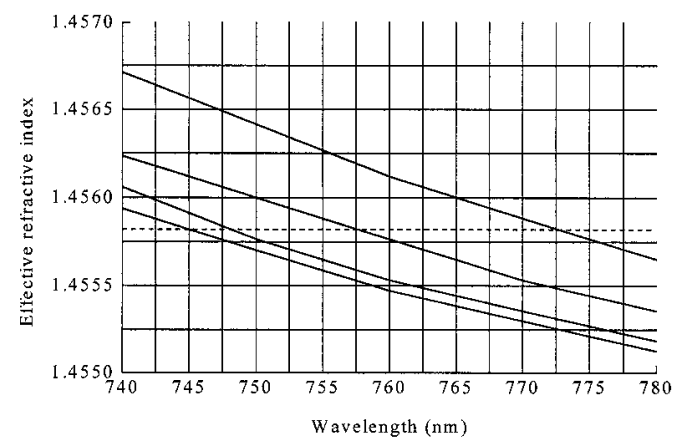

Fig. 12. Theoretical dispersion curves of the overlay waveguide in solutions of, from left to right, $\mathrm{pH} 11.56,11.98,12.60$, and 13.23. The dotted line represents the dispersion of the optical fiber.

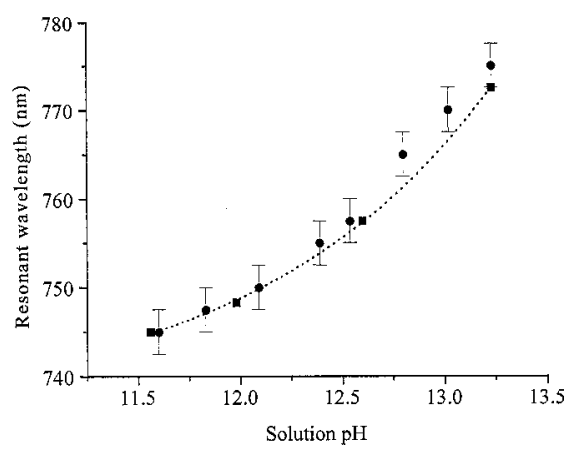

Fig. 13. Plot of measured (circles) and predicted (squares) phasematching wavelength versus ammonia solution $\mathrm{pH}$. refractive index required for phase matching at 745 $\mathrm{nm}$ corresponding to the sensor response to an ammonia solution of $\mathrm{pH}$ 11.6. After we made the calculations it was possible to obtain absolute refractive-index values corresponding to exposure of the overlay material to the $\mathrm{pH}$ values shown in Fig. 10.

\section{Conclusions}

A fiber-optic chemical sensor based on a side-polished single-mode optical fiber, evanescently coupled to a chemically sensitive overlay planar waveguide, has been demonstrated as a $\mathrm{pH}$ sensor. LB deposition has been shown to be an excellent technique for the formation of an overlay waveguide because it allows for fine thickness control. Furthermore, inasmuch as the materials that form the overlay are organic dyes, it might be possible to tailor the response of the materials for specific purposes. Finally, a theoretical model based on Kramers-Kronig relations has been developed that allows the response of the sensor to be predicted and that has shown good agreement with the nonlinear experimental results.

\section{References}

1. R. A. Lieberman, "Recent progress in intrinsic fibre-optic chemical sensing II," Sens. Actuators B 11, 43-55 (1993).

2. H. Tai, H. Tanaka, and T. Yoshino, "Fiber-optic evanescentwave methane-gas sensor using optical absorption for the 3.392- $\mu \mathrm{m}$ line of a He-Ne laser," Opt. Lett. 12, 437-439 (1987).

3. B. Culshaw, F. Muhammad, G. Stewart, S. Murray, D. Pinchbeck, J. Norris, S. Cassidy, M. Wilkinson, D. Williams, I. Crisp, R. Van Ewyk, and A. McGhee, "Evanescent wave methane gas detection using optical fibers," Electron. Lett. 28, 2232-2234 (1992).

4. R. A. Lieberman, L. L. Blyer, and L. G. Cohen, "A distributed fibre optic sensor based on cladding fluorescence," J. Lightwave Technol. 8, 212-220 (1990).

5. D. Flannery, S. W. James, R. P. Tatam, and G. J. Ashwell, "pH sensor using Langmuir-Blodgett overlays on polished optical fibres," Opt. Lett. 22, 567-569 (1997).

6. R. B. Charters, S. E. Staines, and R. P. Tatam, "In-line fiberoptic components using Langmuir-Blodgett films," Opt. Lett. 19, 2036-2038 (1994).

7. V. Ramaswamy, "Propagation in asymmetrical anisotropic film waveguides," Appl. Opt. 13, 1363-1371 (1974).

8. C. D. Hussey and J. D. Minelly, "Optical fibre polishing with a motor-driven polishing wheel," Electron. Lett. 24, 805-807 (1988).

9. O. G. Leminger and R. Zengerle, "Determination of singlemode fiber coupler design parameters from loss measurements," J. Lightwave Technol. LT-3, 864-867 (1985).

10. M. C. Petty, Langmuir-Blodgett films (Cambridge U. Press, Cambridge, England, 1996).

11. R. A. Potyrailo, V. P. Ruddy, and G. M. Hieftje, "KramersKronig analysis of molecular evanescent-wave absorption spectra obtained by multimode step-index optical fibers," Appl. Opt. 35, 4102-4111 (1996).

12. J. I. Steinfeld, An Introduction to Modern Molecular Spectroscopy (MIT, Cambridge, Mass., 1985).

13. E. Kreyszig, "Numerical analysis," in Instructor's Manual for Advanced Engineering Mathematics, E. Kreyszig, ed., 5th ed. (Wiley, New York, 1983), pp. 788-790.

14. A. K. Ghatak, K. Thyagarajan, and M. R. Shenoy, "Numerical analysis of planar optical waveguides using matrix approach," J. Lightwave Technol. LT-5, 660-667 (1987). 